\title{
Correlación de las características antropométricas frente a la capacidad inspiratoria máxima en sujetos hospitalizados
}

\author{
Correlation of anthropometric characteristics against maximum \\ inspiratory capacity in hospitalized subjects
}

\author{
Javier Eliecer Pereira Rodríguez, ${ }^{*}$ Otilio López Flores, ${ }^{\ddagger}$ \\ Sandra Sharon Waiss Skvirsky, Bruno Espinosa Carrasco ${ }^{\ddagger}$
}

\begin{abstract}
Citar como: Pereira RJE, López FO, Waiss SSS, Espinosa CB. Correlación de las características antropométricas frente a la capacidad inspiratoria máxima en sujetos hospitalizados. Acta Med Grupo Angeles. 2021; 19 (3): 340-345. https://dx.doi.org/10.35366/101725
\end{abstract}

\section{Resumen}

Introducción: La antropometría estudia la composición de un organismo mediante la descripción de sus características físicas. Factores como obesidad o desnutrición generan cambios en la movilidad diafragmática, evidenciando que el reposo prolongado y la disminución de la actividad física en sujetos hospitalizados se asocian a mayores complicaciones pulmonares. Material y métodos: Se realizó una investigación de tipo experimental correlacional a través de variables cuantitativas, seguimiento longitudinal y cronología prospectiva, desarrollando un análisis de media, mediana, desviación estándar, asimetría, percentil y correlación de Pearson en el Hospital Ángeles Lomas. Los análisis fueron con nivel de significancia de $p<0.050$ (IC 95\%). Resultados: La muestra de estudio fue de 65 pacientes (H: 31 vs. M: 34), edad promedio de $57 \pm 17$. Asimismo, el análisis de correlación de Pearson en medidas antropométricas frente a la capacidad inspiratoria máxima demostró correlación baja en peso $\left(y=0.007 x+64.951, R^{2}\right.$ $=0.0805)$, talla $\left(y=5 E-05 x+1.5801, R^{2}=0.1548\right)$, IMC $(y=$ $\left.0.0009 x+25.783, R^{2}=0.018\right)$ y circunferencia abdominal $(y=$ $\left.0.0002 x+102.2, R^{2}=0\right)$. Conclusiones: Sujetos con mayor índice de masa corporal y de sexo masculino poseen mejores parámetros en la capacidad inspiratoria.

Palabras clave: Antropometría, hospitalización, capacidad inspiratoria.

\section{Abstract}

Introduction: Anthropometry studies the composition of an organism describing its physical characteristics. Factors such as obesity or malnutrition generate changes in diaphragmatic mobility, showing that prolonged rest and decreased physical activity in hospitalized subjects are associated with greater pulmonary complications. Material and methods: A correlational experimental research was carried out through quantitative variables, longitudinal follow-up and prospective chronology, developing an analysis of mean, median, standard deviation, asymmetry, percentile and Pearson's correlation at Hospital Ángeles Lomas, the analyzes were with a significance level of $p<0.050(\mathrm{Cl} 95 \%)$. Results: The study sample was 65 patients ( $H: 31$ vs $W: 34)$, mean age of $57 \pm 17$. likewise, Pearson's correlation analysis in anthropometric measurements versus maximum inspiratory capacity showed a low correlation in weight $\left(y=0.007 x+64.951, R^{2}=0.0805\right)$, height $(y=5 E-05 x$ $\left.+1.5801, R^{2}=0.1548\right), B M I\left(y=0.0009 x+25.783, R^{2}=0.018\right)$ and abdominal circumference $\left(y=0.0002 x+102.2, R^{2}=0\right)$. Conclusions: Male subjects with a higher body mass index, have better parameters in inspiratory capacity.

Keywords: Anthropometry, hospitalization, inspiratory capacity.
* Fisioterapeuta, Especialista en Rehabilitación Cardiopulmonar, Magíster en Cuidados Paliativos, Doctorando en Fisiología. Centro de Estudios e Investigación FISICOL. Bogotá, Colombia.

‡ Fisioterapeuta. Centro Integral de Rehabilitación, S.C. en el Hospital Ángeles Lomas. Huixquilucan, México.

$\S$ Fisioterapeuta. Liderazgo de Organizaciones. Directora de Fisioterapia y Rehabilitación del Centro Integral de Rehabilitación, S.C. en el Hospital Ángeles Lomas. Huixquilucan, México.
Correspondencia:

Javier Eliecer Pereira Rodríguez

Correo electrónico: jepr87@hotmail.com

Aceptado: 29-09-2020.

www.medigraphic.com/actamedica 


\section{INTRODUCCIÓN}

La antropometría es una ciencia que estudia el tamaño, proporción, maduración, forma, composición corporal y funciones generales del organismo con el objetivo de describir las características físicas, evaluar y monitorizar el crecimiento, nutrición y los efectos de la actividad física. ${ }^{1}$ Se basa en cuatro pilares básicos: las medidas corporales, estudio del somatotipo, estudio de la proporcionalidad y el estudio de la composición corporal. Actualmente, la antropometría se aplica en diversas áreas de la medicina para estudiar las enfermedades y anomalías que afectan las dimensiones del cuerpo humano, ya que éstas varían de un individuo a otro según su edad, sexo, raza, nivel socioeconómico, entre otros factores. ${ }^{2}$

Dentro de sus variables, el índice de masa corporal (IMC), circunferencia abdominal, índice cintura/cadera (ICC), mediciones corporales de tipo peso, estatura y pliegues grasos se utilizan en la antropometría por ser un método fácil, económico y no invasivo para obtener información sobre la presencia de desnutrición, sobrepeso, obesidad y cofactores de riesgo. ${ }^{3}$ Datos de la Organización Mundial de la Salud (OMS) determinan que un índice de masa corporal normal corresponde a valores de 18.5$24.9 \mathrm{~kg} / \mathrm{m}^{2}$, sobrepeso $\geq 25 \mathrm{~kg} / \mathrm{m}^{2}$ y obesidad $\geq 30 \mathrm{~kg} / \mathrm{m}^{2}$, mientras que parámetros de circunferencia abdominal máximo saludable menor de $88 \mathrm{~cm}$ en las mujeres y 102 $\mathrm{cm}$ en los hombres. ${ }^{4}$

Por tanto, se ha encontrado una relación con la dinámica respiratoria y el exceso de grasa corporal que recubre el tórax y el abdomen, que demuestra que se genera una restricción en la movilidad diafragmática y en la parrilla costal, lo que produce cambios significativos en la dinámica y funcionalidad del sistema ventilatorio que desencadenan un patrón respiratorio restrictivo. ${ }^{5}$ La obesidad se asocia a distintas enfermedades y se comporta como un importante factor de riesgo cardiovascular, mismo que tiene un reconocido impacto en la prevalencia y el pronóstico de algunas enfermedades respiratorias como asma, síndrome de apneas-hipoapneas del sueño, neumonía, complicaciones respiratorias perioperatorias y periodos prolongados de estancia hospitalaria. ${ }^{6}$

En consecuencia, los trastornos metabólicos alteran de manera directa las propiedades del tórax y los pulmones mediante la acumulación de grasa en el mediastino, el abdomen y la cavidad torácica, lo que puede generar una hipertrofia muscular secundaria a un mayor trabajo respiratorio que supone la sobrecarga mecánica. ${ }^{7}$ Por otra parte, se han descrito casos de infiltración de grasa en músculos inspiratorios que genera una disfunción muscular; por último, cambios en la configuración del tórax que dan lugar a una inadecuada relación longitud-tensión y como resultado dificultad en la obtención de presiones inspiratorias adecuadas. ${ }^{8}$

Por otra parte, la afectación del estado nutricional en sujetos de infrapeso es una de las comorbilidades más comunes en patologías pulmonares crónicas. La pérdida de peso y desnutrición están asociadas a un peor pronóstico de la enfermedad debido a factores como el desequilibrio energético (por aumento del gasto energético basal [GEB]), desequilibrio entre síntesis y catabolismo proteico, ingesta alimentaria reducida, atrofia muscular por baja actividad física, estado de inflamación sistémico, estrés oxidativo así como otros factores más específicos como la susceptibilidad genética, la insuficiencia hormonal, complicaciones infecciosas y el efecto térmico. ${ }^{9}$

En consecuencia, dichas alteraciones generan cambios en los volúmenes y capacidades pulmonares, como es el caso de la capacidad inspiratoria máxima que hace referencia al volumen de gas que puede ser introducido en el pulmón con un esfuerzo inspiratorio máximo que está dado por la suma del volumen corriente y volumen de reserva inspiratorio, mientras que la capacidad pulmonar total se define como el máximo volumen de gas que pueden contener los pulmones (aproximadamente 5,800 mL), mismos que se verán limitados en diferentes situaciones por el proceso de expansión pulmonar. ${ }^{10}$

Por último, se ha evidenciado que la estrecha relación que presenta el reposo prolongado en cama y la disminución de la actividad física en sujetos hospitalizados se asocia a diferentes trastornos metabólicos, representando un factor predisponente al desarrollo o al agravamiento de ciertas condiciones patológicas relacionadas en mayor medida con el sistema cardiovascular y respiratorio en el cual destacan neumonías, derrame pleural, alteraciones morfológicas del parénquima, flujo aéreo y volúmenes pulmonares así como afectación musculoesquelética, lo que conlleva a un aumento de los días de hospitalización. ${ }^{11}$

Dicho lo anterior, surge como pregunta de investigación: ¿Cuál es la correlación de las medidas antropométricas frente a la capacidad inspiratoria máxima en sujetos hospitalizados? Buscando una posible respuesta a esta pregunta, el objetivo principal de la presente investigación es conocer la correlación de las medidas antropométricas (talla, peso, CA e IMC) frente a la capacidad inspiratoria máxima en sujetos hospitalizados.

\section{MATERIAL Y MÉTODOS}

Este estudio se realizó a partir de una investigación de tipo experimental correlacional a través de variables cuantitativas mediante un seguimiento longitudinal y cronología prospectiva en una población de 65 pacientes hospitalizados del Hospital Ángeles Lomas, misma que estuvo 
representada por sujetos mayores de 18 años de edad que fueron ingresados como interconsulta al Servicio de Fisioterapia y Rehabilitación del mismo hospital. Los pacientes hemodinámicamente inestables, con pérdida del estado de conciencia, sedados, intubados, nula cooperación u otros procesos que pudiesen alterar las variables controladas 0 la integridad del sujeto fueron excluidos del estudio.

Por otro lado, los criterios de inclusión fueron pacientes con componente pulmonar, neumonía, derrame pleural, postoperatorio de cirugía abdominal, antecedentes de enfermedad pulmonar obstructiva crónica (EPOC), obesidad así como alteraciones cardiacas o terapia pulmonar para prevenir infecciones nosocomiales por estancia prolongada de hospitalización.

Los procedimientos para la obtención de las mediciones antropométricas, la capacidad inspiratoria máxima así como el uso de la información se explicaron a cada uno de los participantes y/o familiar a cargo. Se procedió a la recolección de la información básica y datos personales de cada uno de los participantes a partir de una base de datos elaborada por los autores de este estudio. Se usó la información proporcionada en el expediente clínico del paciente así como una doble verificación de la misma para la determinación de la talla y peso de cada participante.

La circunferencia abdominal se determinó mediante el uso de una cinta métrica de $150 \mathrm{~cm} \mathrm{60",} \mathrm{posicionando} \mathrm{al}$ sujeto en bipedestación o posición supina, brazos relajados a cada lado del mismo colocando la cinta métrica entre la décima costilla y la cresta iliaca como referentes por cada individuo tras una espiración fisiológica sin presión de contacto cinta-piel ${ }^{12}$ Asimismo, el IMC se estableció con base en la fórmula utilizada de peso en kilos dividido entre la estatura del paciente al cuadrado $(\mathrm{IMC}=$ peso $(\mathrm{kg}) /$ altura $\left.(\mathrm{m})^{2}\right)$ propuesta por Quetelet y su interpretación por la Organización Mundial de la Salud, ${ }^{13}$ registrando sus valores en la base de datos ya establecida.

Por último, la capacidad inspiratoria máxima se obtuvo mediante tres mediciones realizadas con un inspirómetro volumétrico de $5,000 \mathrm{~mL}$, en el cual se le pedía al paciente mantener una postura sedente y sostener el dispositivo, poner la boquilla del inspirómetro en la boca asegurando hacer un buen sello con los labios, posteriormente se realizaba una exhalación hasta llegar a volumen residual seguido de una inhalación lenta y profunda tanto como le fuera posible, obteniendo un valor pico (máxima cantidad de aire que se obtuvo en los tres intentos) y un valor promedio, el cual era medido por la suma de los intentos y dividido entre tres, el inspirómetro volumétrico se utilizó como dispositivo de medición por su fácil acceso, cuantificación inspiratoria en $\mathrm{mL}$, bajo costo, reproducibilidad y fácil dominio del terapeuta.

Dichas mediciones se realizaron de manera longitudinal mediante la obtención de las medidas antropométricas en el ingreso y egreso del paciente, así como una medición diaria de la capacidad inspiratoria máxima con el mismo esquema de tres repeticiones diarias para la obtención de pico máximo y promedio inspiratorio.

\section{Análisis estadístico}

Se desarrolló el análisis de las variables cuantitativas media, mediana, desviación estándar, asimetría, per-

Tabla 1: Características de la población de estudio.

\begin{tabular}{lccccc} 
Características & Hombres & Mujeres & Total & Mediana & Moda \\
\hline Edad & $51 \pm 18$ & $62 \pm 14$ & $57 \pm 17$ & 58 & 72 \\
Sexo & 31 & 34 & 65 & - & - \\
Antropometría & & & & & 74 \\
$\quad$ Peso & $88 \pm 23$ & $66 \pm 11$ & $77 \pm 20$ & 1.67 & 1.63 \\
$\quad$ Talla & $1.7 \pm 0.13$ & $1.6 \pm 0.07$ & $1.67 \pm 0.11$ & 101 & 103 \\
$\quad$ Circunferencia abdominal & $109 \pm 31$ & $97 \pm 20$ & $103 \pm 26$ & 26.6 & 2.97 \\
IMC & $29 \pm 5.9$ & $25 \pm 4.3$ & $27 \pm 5.5$ & & \\
Estado civil (\%) & & & & & \\
$\quad$ Casado/a & 81.00 & 73.00 & & & 2,000 \\
$\quad$ Soltero/a & 19.00 & 12.00 & & & \\
$\quad$ Viudo/a & 0 & 15.00 & & & \\
Función pulmonar & & & & & \\
$\quad$ Capacidad inspiratoria máxima & $1,946.7$ & $1,501.4$ & $1,713.8$ & &
\end{tabular}




\begin{tabular}{lccrr}
\hline \multicolumn{2}{c}{ Tabla 2: Capacidad inspiratoria según índice de masa corporal. } \\
\hline & Infrapeso & Normopeso & Sobrepeso & Obesidad \\
\hline $\begin{array}{l}\text { Pacientes } \\
\text { Valor promedio de inspirómetro }(\mathrm{mL})\end{array}$ & 13 & 20 & 23 & 19 \\
\hline
\end{tabular}

centil y coeficiente de correlación de Pearson. Por otro lado, se realizó la gráfica de dispersión de variables de días de hospitalización, variaciones de circunferencia abdominal en ingreso y egreso hospitalario así como su correlación respecto a la capacidad inspiratoria máxima frente a las medidas antropométricas de los sujetos hospitalizados en el Hospital Ángeles Lomas. Estos datos se determinaron mediante filtración de datos obtenidos en el programa de Office: Microsoft Excel 16.0, todos los análisis fueron con un nivel de significancia de $p<$ 0.050 (IC 95\%).

\section{RESULTADOS}

En la presente investigación correlacional ingresaron 173 pacientes, de los cuales 108 fueron descartados tras aplicar los criterios de inclusión y exclusión en la muestra inicial. Luego de ello, se logró obtener así una muestra final de 65 pacientes (H: 31 vs. $\mathrm{M}$ : 34).

Dicho esto, es importante conocer que la edad promedio de la muestra total fue de $57 \pm 17$, y se resalta que el sexo femenino fue de mayor edad en comparación con el sexo masculino $(H: 51 \pm 18$ vs. M: $62 \pm 14)$. Dato contrario a los encontrados en el peso ( $\mathrm{H}: 88 \pm 23$ vs. M: $66 \pm 11)$, talla $(\mathrm{H}: 1.7 \pm 0.13$ vs. $\mathrm{M}: 1.6 \pm 0.07)$, índice de masa corporal $(\mathrm{H}: 29 \pm 5.9$ vs. $\mathrm{M}: 25 \pm 4.3)$ y circunferencia abdominal $(\mathrm{H}: 109 \pm 31$ vs. M: $97 \pm 20)$ donde claramente el sexo masculino presentó datos mayores frente al sexo femenino (Tabla 1). Seguidamente, es necesario conocer las características antropométricas desde el punto de vista del IMC de los participantes para más adelante poder correlacionar las variables del estudio. Para ello, describimos que tres participantes (4.62\%) tenían infrapeso, 20 (30.77\%) normopeso, 23 (35.38\%) y 19 (29.23\%) con sobrepeso y obesidad respectivamente (Tabla 2).

Por otra parte, al realizar el análisis respectivo de correlación de la $r$ de Pearson en las medidas antropométricas frente a la capacidad inspiratoria máxima logramos encontrar que existe una correlación baja para el peso (y $\left.=0.007 \times+64.951, R^{2}=0.0805\right)$, talla $(y=5 E-05 \times+$ $\left.1.5801, R^{2}=0.1548\right)$, IMC $\left(y=0.0009 \times+25.783, R^{2}\right.$ $=0.018)$ y circunferencia abdominal $(y=0.0002 \times+$ 102.2, $\mathrm{R}^{2}=0$ ) (Figuras 1 y 2).

\section{DISCUSIÓN}

La presente investigación intentó demostrar la relación entre las medidas antropométricas y la capacidad inspiratoria máxima al ingreso y egreso hospitalario debido a la importancia de ambas variables en condiciones clínicas mediante un seguimiento longitudinal de pacientes hospitalizados. Daymond I y colaboradores ${ }^{14}$ en su estudio mencionan que las modificaciones en el estado nutricional como la desnutrición o el infrapeso tienen efectos adversos en el sistema respiratorio: dañan el movimiento ventilatorio y disminuyen la fuerza de los músculos respiratorios; asimismo, observaron una reducción de la presión espiratoria máxima en 59\%, y de la presión inspiratoria en 43\%; en este estudio los participantes con infrapeso mostraron una capacidad inspiratoria máxima disminuida $y=21.531 \times R^{2}=-0.35$ con una correlación de Pearson baja debido a la pequeña muestra de sujetos en esta categoría; sin embargo, los valores obtenidos de $\leq 1,883 \mathrm{~mL}$ en inspirometría volumétrica se asociaron a las enfermedades de base así como a procedimientos realizados durante su estancia hospitalaria.

Por otra parte, Valenza $M$ y colaboradores ${ }^{15}$ en su estudio confirman que factores como la edad, talla y procesos de envejecimiento propician una pérdida de fuerza muscular, capacidad cardiovascular y una reducción de la movilidad articular, generando como resultando una disminución de la capacidad pulmonar, y determinaron que el sexo masculino posee mayores valores de función pulmonar en comparación con el sexo femenino aun con rangos iguales o semejantes de peso, edad, distribución de grasa corporal y porcentaje de masa muscular, mismos resultados que se asemejan a los descritos en este estudio respecto a la capacidad inspiratoria medida en ambos sexos.

Miranda M y Muñoz $\mathrm{R}^{16}$ reportan en su estudio la relación de variables de peso e IMC respecto a la distribución de la grasa corporal, explorando una reducción de la función pulmonar directamente proporcional al incremento de la obesidad abdominal, en la cual muestran reducciones del volumen espiratorio forzado en un segundo por cada kilogramo ganado de peso, de entre 13.9 y $23 \mathrm{~mL} / \mathrm{kg}$ así como datos inferiores a los esperados en la capacidad inspiratoria correspondiente a los individuos estudiados. 
Figura 1: Correlación de la capacidad inspiratoria en sujetos con obesidad.

$-\quad$ INS Lineal (INS)

Índice de masa corporal

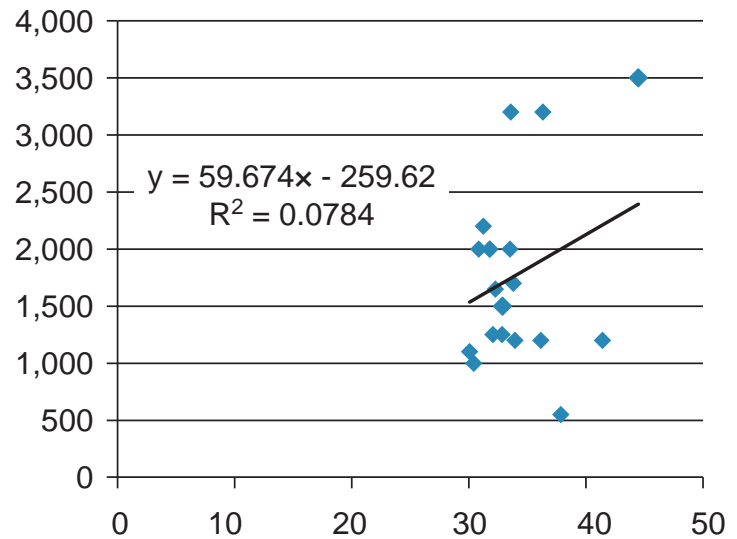

Peso

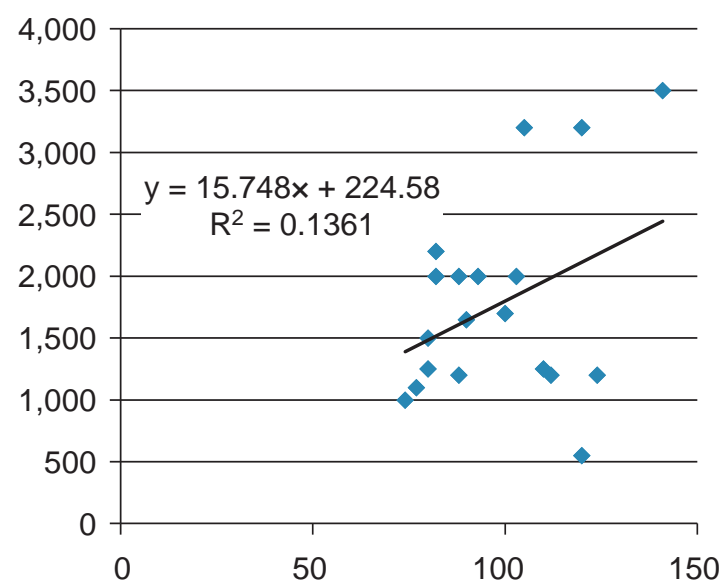

Edad

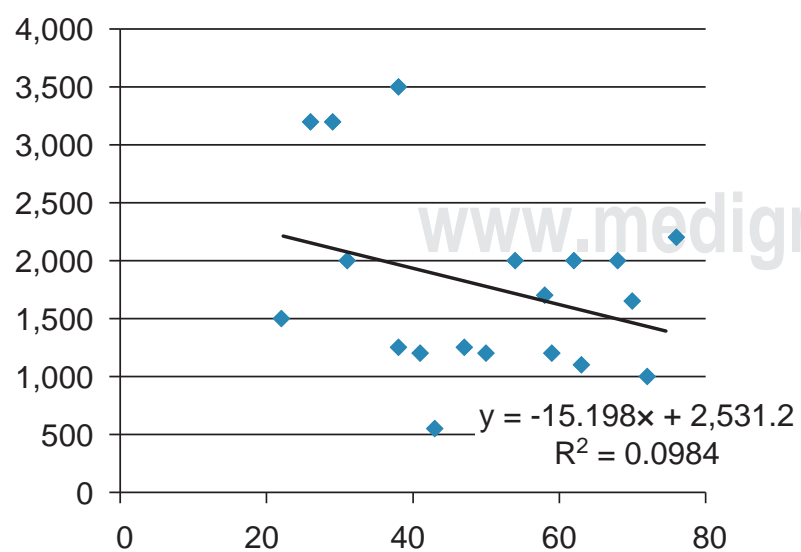

Figura 2: Correlación de la capacidad inspiratoria según la antropometría.

Sobrepeso (IMC)

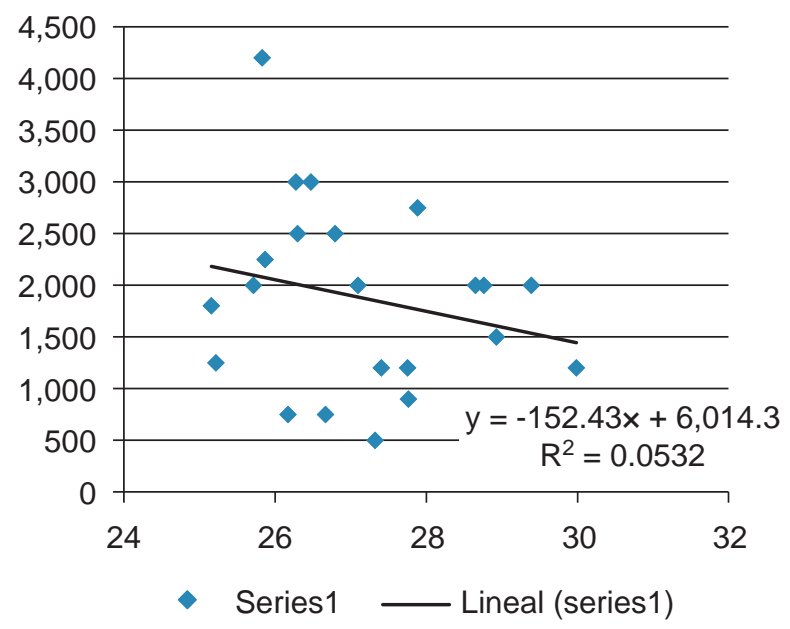

Normopeso (IMC)

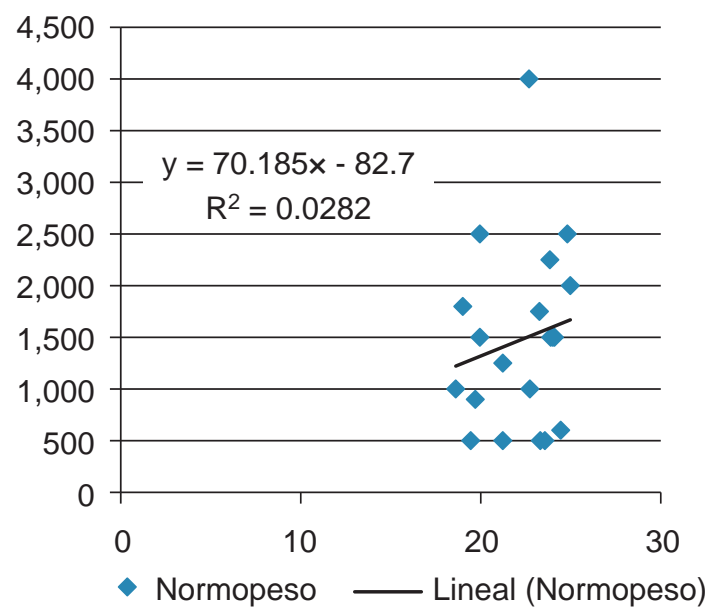

Infrapeso (IMC)

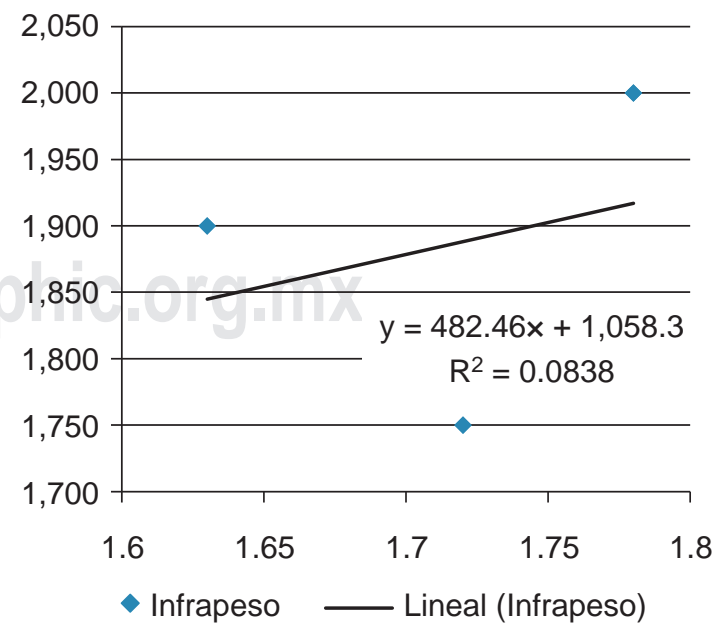


Sin embargo, en el presente estudio no se encontró relación peso e IMC respecto a la capacidad inspiratoria máxima, evidenciando que la población con mayor porcentaje graso no presenta cambios significativos en la capacidad pulmonar en comparación con sujetos normopeso, incluso se demostró que sujetos con mayor IMC presentaron mejores resultados en la prueba de inspirometría volumétrica.

Datos relevantes de la investigación presentada generan diferencias en cuanto a lo reportado en investigaciones previas y esto se asocia a múltiples causas, probablemente los valores de capacidad inspiratoria máxima respecto a las medidas antropometrías encontradas en la población de este estudio se deben a diferentes factores, entre los que destacan sus comorbilidades, antecedentes heredofamiliares y siendo principalmente la enfermedad de base (enfermedades pulmonares, obesidad, enfermedades autoinmunes así como procedimientos quirúrgicos realizados).

\section{CONCLUSIONES}

Con base en los resultados obtenidos, y dando respuesta a la pregunta de investigación planteada, se concluye que existe una correlación baja entre las medidas antropométricas y la capacidad inspiratoria máxima en sujetos hospitalizados, además se concluyó que sujetos con mayor IMC y de sexo masculino poseen mejores parámetros en inspirometría volumétrica, de la misma manera se evidenció que la no estandarización de enfermedades hospitalarias de la población en estudio genera una disminución de la corrección de las variables debido a múltiples factores como enfermedades pulmonares crónicas, cirugías abdominales, procesos oncológicos, dolor, entre otros.

\section{REFERENCIAS}

1. Carmenate LM, Moncada CFA, Waldermar EBL. Manual de medidas antropométricas. Costa rica: SALTRA; 2014.

2. Nariño R, Becerra A, Hernandez A. Anthropometry. Comparative analysis of technologies for the capture of anthropometric dimensions. Revista EIA. 2016; 13 (26): 47-59.

3. Cedeño MR, Castellanos GM, Benet RM, Mass SL, Mora HC, Parada AJ. Indicadores antropométricos para determinar la obesidad, y sus relaciones con el riesgo cardiometabólico: cifras alarmantes. Rev Finlay. 2015; 5 (1): 12-23.
4. Organización Mundial de la Salud. Obesidad y sobrepeso. [Acceso 3 de marzo de 2020] Disponible en: https://www.who.int/es/newsroom/fact-sheets/detail/obesity-and-overweight

5. Rodríguez-Valdés S, Donoso-Riveros D, Sánchez-Peña E, MuñozCofré $\mathrm{R}$, Conei $\mathrm{D}$ del-Sol $\mathrm{M}$ et al. Uso del índice de masa corporal y porcentaje de grasa corporal en el análisis de la función pulmonar. Int J Morphol. 2019; 37 (2): 592-599. doi: 10.4067/s071795022019000200592.

6. Carpió C, Santiago A, García de Lorenzo A, Álvarez-Sala R. Función pulmonar y obesidad. Nutr Hosp. 2014; 30 (5): 1054-1062. Disponible en: https://dx.doi.org/10.3305/nh.2014.30.5.8042

7. Rabec C, Lucas Ramos P, Veale D. Complicaciones respiratorias de la obesidad. Arch Bronconeumol. 2011; 47 (5): 252-261. doi: 10.1016/j.arbres.2011.01.012.

8. Thomaz T, Rufino R, Costa $\mathrm{C}$ et al. Obesidad: complicaciones sistémicas y pulmonares, anomalías bioquímicas y deterioro de la función pulmonar. Medicina Respiratoria Multidisciplinaria. 2016; 11 (28): 17-19.

9. Benito Martínez MDP, La Serna Infantes JE, Guarro Riba M, Morera Ingles M, Camere Colarossi DM, Camere Torrealva MA. Nutritional and functional state of patients with chronic obstructive pulmonary disease: effects of oral nutritional supplementation (OFOS study). Nutr Hosp. 2017; 34 (4): 776-783.

10. García RF, Lores y Blas RV. Evaluación funcional respiratoria (obstrucción y atrapamiento). Arch Bronconeumol. 2007; 43 Supl 3: 8-14.

11. Ibarra Cornejo JL, Fernández Lara MJ, Aguas Alveal EV, Pozo Castro AF, Antillanca Hernández B, Quidequeo Reffers DG. Efectos del reposo prolongado en adultos mayores hospitalizados. An Fac Med. 2017; 78 (4): 439-444. Disponible en: https://dx.doi.org/10.15381/ anales.v78i4.14268

12. McCarthy HD, Ashwell M. A study of central fatness using waistto-height ratios in UK children and adolescents over two decades supports the simple message- 'keep your waist circumference to less than half your height'. Int J Obes (Lond). 2006; 30 (6): 988-992.

13. WHO Expert Consultation. Appropriate body-mass index for Asian populations and its implications for policy and intervention strategies. Lancet. 2004; 363 (9403): 157-163.

14. García ADI, Trujillo-Hernández B, González-Sánchez R, Vásquez C, Trujillo-Magallón M, Trujillo-Magallón E. Correlation between nutritional status and spirometric parameters in adolescents in Colima, Mexico. Archivos de Medicina. 2016; 12 (3): 1-5.

15. Valenza MC, Martin LM, Botella ML, Castellote YC, Revelles FM, Serrano MG et al. Pulmonary function, the physical factors that determine it and its importance for the physiotherapist. Revista Iberoamericana de Fisioterapia y Kinesiología. 2011; 14 (2): 83-89.

16. Miranda MM, Muñoz RC. Reliability and validity of incentive spirometer in inspiratory capacity measurement. REEM. 2014; 1 (1): 27-31.

Conflicto de intereses: Los autores declaran no tener ningún tipo de conflicto de intereses. 